\title{
Trailing TRAIL Resistance for Targeted Cancer Therapy
}

\author{
Ngai Siew Ching* and Wong How Ming Sonia \\ Faculty of Science, University of Nottingham Malaysia Campus, Malaysia \\ Received: April 09, 2018; Published: April 20, 2018
}

*Corresponding author: Ngai Siew Ching, School of Biosciences, Faculty of Science, University of Nottingham Malaysia Campus, 43500 Semenyih, Selangor, Malaysia, Email: Eunice.Ngai@nottingham.edu.my

\begin{abstract}
Tumour necrosis factor (TNF)-related apoptosis-inducing ligand (TRAIL) is a promising anti-cancer agent due to its selective killing of cancer cells, sparing the vital normal cells. Despite its great potential in cancer treatment, its clinical application has been hampered by the cancer resistance. Various strategies have been developed to overcome resistance towards TRAIL-induced apoptosis which include the combinational therapy of TRAIL with epigenetic drugs, chemotherapy drugs and the autophagy inhibitors. To heighten the therapeutic outcome, the personalized medicine based on biomarker screening could serve as a novel strategy in tailoring the combinational therapy of TRAIL and sensitizer for targeted cancer therapy in the near future.
\end{abstract}

Abbreviations: TNF: Tumour Necrosis Factor; TRAIL: Tumour Necrosis Factor Related Apoptosis-Inducing Ligand; DISC: Death Inducing Signalling Complex; FADD: Fas Associated Death Domain

\section{Introduction}

Apoptosis is an orchestrated cell death that occurs in physiological condition. The loss of balance between cell division and cell death leads to pathological condition such as cancer [1]. Despite remarkable advances in the understanding the cancer biology and the development of novel diagnostic and therapeutic strategies, cancer still remains one of the major causes of death [2]. The lack of specificity of the current cancer therapy sparked an urgency to unravel a targeted cancer therapy. Tumour necrosis factor (TNF)-related apoptosis-inducing ligand (TRAIL), a member of TNF cytokine superfamily is a promising anti-cancer agent due to its selective targeting on TRAIL-R1 (DR4) and TRAIL-R2 (DR5) expressed by the cancer cells, sparing the normal cells [3].

The binding of TRAIL to TRAIL-R1 and/or TRAIL-R2 results in receptor trimerization, Fas-associated death domain (FADD) recruitment, death inducing signalling complex (DISC) assembly and caspase cascade activation, thereby inducing extrinsic apoptosis pathway in type I cells $[2,4]$. As a consequence of TRAIL DISC activation, the induction of intrinsic apoptosis pathway is required in type II cells for efficient TRAIL-induced apoptosis [5,6]. Despite the specificity of TRAIL towards cancer cells, the validity of TRAIL for targeted cancer therapy has yet to be established. One of the hurdles which hampers its clinical application is TRAIL resistance. The resistance of TRAIL-induced apoptosis and the strategies that have been explored to overcome TRAIL resistance in developing an improved targeted cancer therapy will be reviewed.

\section{Resistance}

In line with TRAIL targeted cancer therapy, a recombinant form of human soluble TRAIL (Apo2L.0 or AMG-951/Dulanermin) and agonistic antibodies that specifically target TRAIL-R1 and TRAIL-R2 have been brought from bench to clinical trials [7]. TRAIL therapy has a major limitation as a large number of the cancer develops resistance towards TRAIL [8], either intrinsically resistant or acquired during the course of treatment [9-11]. The mechanisms which inhibit apoptosis execution can be divided into:

a. Disrupted balance between pro-apoptotic and antiapoptotic proteins, resulting in dysregulated apoptosis in the affected cells which could be due to over-expression of antiapoptotic proteins (such as Bcl-2, Bcl-xL, cIAPs, XIAP and c-FLIP) [12] or under-expression of pro-apoptotic proteins (such as Bid)[1];

b. Reduced caspase function which leads to decreased apoptosis [1]; and

c. Impaired death receptor signalling which can be caused by down-regulation of receptor surface expression (mutations and epigenetic silencing) $[13,14]$, clathrin-related endocytosis of TRAIL-death receptor, accumulation of autophagosome, coexistence of decoy receptor, post-translation modifications (down-regulation of fucosylation and O-linked glycosylation and up-regulation of $\mathrm{N}$-linked glycosylation) leads to evasion of extrinsic apoptosis pathway [1,4,15-17]. 
The sensitization of cancer cells towards TRAIL-induced apoptosis tackling the above-mentioned mechanisms will be reviewed. The other mechanisms of TRAIL resistance include aberrant protein synthesis, protein misfolding, ubiquitin regulated death receptor expression, metabolic pathways and metastasis [8]. However, the detailed resistance mechanism is not well elucidated and it is not known whether different types of cancer undergo TRAIL resistance through similar or specific mechanisms.

\section{Sensitization of Cancer Cells Towards TRAIL-Induced Apoptosis}

Based on its value as a targeted anti-cancer therapeutic agent, TRAIL is worth to be further investigated despite the various defensive resistance mechanisms pitched by different cancer cells to safeguard themselves from the devastating TRAIL-induced apoptosis fate. Recent advances in pre-clinical research on potential combination of TRAIL with other established drugs in sensitizing cancer cells towards TRAIL anti-cancer effects have shed light that these TRAIL resistance mechanisms can indeed be tweaked towards the favourable outcome against cancer cells.

As aforementioned, the skewed balance between pro-apoptotic and anti-apoptotic proteins can render cancer cells to be TRAIL-resistant. For instance, over expression of Bcl-2 and associated anti-apoptotic proteins Bcl-xL, Mcl-1, A1/Bf1 and Bcl-w have been commonly associated with resistance of cancer cells such as pancreatic, ovarian, lymphoma, multiple myeloma, lung adenocarcinoma, prostate adenocarcinoma against the chemotherapy-mediated cell death in the intrinsic apoptotic pathway [18]. Likewise, in the case of TRAIL-induced apoptosis axis, every stage of the extrinsic apoptotic pathway like its cognate receptors, TRAIL-R1 and TRAIL-R2, trimerization, FADD recruitment, DISC assembly, caspase activation and eventual engagement of mitochondrial intrinsic pathway can potentially be harnessed by cancer cells towards the benefit of their survival.

\section{Epigenetic drugs}

Epigenetic dysregulation of gene expression by DNA methylation and histone deacetylation lead to carcinogenesis $[19,20]$. Epigenetic drugs are useful in this sense because cancer cells have, in many instances, acquired TRAIL resistance via epigenetic DNA modifications which have silenced one or a few of the aforementioned pro-apoptotic members of the extrinsic or intrinsic pathway. For instance, the silenced caspass- 8 caused by the hypermethylation of the promoter in invasive neuroblastoma cell line could be re-expressed by the demethylating agent, 5 '-aza2'deoxycytidine, and it has sensitized invasive NB cells towards TRAIL-induced cell death [21]. Another similar study on small cell lung carcinoma cell line which is highly refractory towards FasL and TRAIL-induced apoptosis due to reduced levels of caspase- 8 and TRAIL-R1 mRNA has also displayed improved sensitivity towards cell death by the combined treatment of 5 '-aza-2-deoxycytidine and interferon gamma [22].

In addition, the other epigenetically modulated members of the TRAIL-induced apoptotic pathway such as TRAIL-R1, TRAIL-R2, caspase- 3 and -8 as well as the pro- and anti-apoptotic proteins have also been successfully manipulated by demethylating agents (such as decitabine, 5-aza-2'deoxycytidine and zebularine) and histone deacetylase inhibitors (such as suberoyl anilidehydroxamic, valproic acid and Entinostat) [8,13,21,23-29] either as single agents or combination [30]. Besides, an increase in fucosylation levels has been reported in many cancer cell lines treated by zebularine, overcoming the resistance of TRAIL-induced apoptosis [26]. These finding revealing the appealing potential of epigenetic drugs as sensitizers of TRAIL-induced apoptosis in cancer cells.

\section{Chemotherapy Drugs}

Accruing evidence suggests that TRAIL resistance mechanisms can be vastly different across various cancer types and the current elucidation of these mechanisms is only the tip of the iceberg. Before the specific targeting against resistance towards the TRAIL-induced extrinsic apoptotic pathway is illuminated, chemotherapy acts as a potential sensitizer towards TRAIL-induced apoptosis by its engagement of the mitochondrial pathway [11]. The chemotherapy drugs include paclitaxel, carboplatin, bevacizumab, doxorubicin, decitabine [8]. The sensitization mostly involves members of the intrinsic apoptotic pathway coupling it to the TRAIL-induced extrinsic apoptosis cascade like Bax [31-33] and Bak [34]. In addition, the sensitization occurs either through up regulation of DR4 or DR5, caspase-3, or through down regulation of Bcl-xLor c-FLIP has also been reported [11,35]. All these data point us towards a larger horizon in tackling TRAIL resistance via multiple unprecedented pathways with the combination of chemotherapy and TRAIL. Indeed, the combination of various chemotherapy drug and recombinant human TRAIL and TRAIL-R1 or TRAIL-R2 monoclonal antibodies is evaluated at the clinical trials [36].

\section{Autophagy Inhibitors}

Alike apoptosis, autophagy is a catabolism of cellular constituents to maintain cellular homeostasis which is also stimulated in response to pathological conditions [37]. Despite its pro-apoptotic effect in some cases [38,39], tumour-associated autophagy has been implicated in increased of cell proliferation and chemo-resistant [40-44]. Since TRAIL is implicated in autophagy induction in different cancers [4], the inhibition of autophagy by pharmacological inhibitors such as mammalian target of rapamycin complex 1 and by silencing the Beclin-1 or ATG7 genes, has sensitized TRAIL-resistant cells to TRAIL-induced apoptosis $[45,46]$.

\section{Other Sensitizers}

In addition to the aforementioned sensitizers, the other sensitizers include multi-kinase inhibitor sorafenib, smac mimetics and protease inhibitor [10]. For instance, sorafenib was also shown to directly induce the rapid dissipation of mitochondrial membrane potential and reactive oxygen species production which subsequently enabled TRAIL to activate caspase-8 in renal cell carcinoma [47]. On the other hand, type II carcinoma cells which are dysfunctional in the engagement of amplification loop from the mitochondrial death signalling have recently been switched to 
TRAIL-induced type I apoptosis activation through XIAP inhibition by SMAC mimetic LBW-242 and Bortezomib [48].

\section{Conclusion and Future Prospects}

The cancer resistance to TRAIL has spurred the combinational therapy to restore apoptosis sensitivity [29]. The combinational therapy of TRAIL with either chemotherapy drugs, epigenetic modulators or autophagy inhibitors has shown to sensitize the TRAIL resistant cancer cells towards TRAIL-induced apoptosis via various molecular mechanisms. To heighten the therapeutic efficacy, the combinational therapy approach could be tailored with the screening of patients with potent biomarkers that predict the responsiveness to TRAIL therapy [12]. These biomarkers include high expression of $\mathrm{N}$-acetylgalctosaminyltransferase-14 (GALNT14) (O-glycosylation initiating enzyme), the presence of at least one functional receptor and low or absent of Six1 (a homeoprotein identified as a novel mediator of TRAIL resistance) expression [9]. Besides, the search for novel biomarkers that predict the responsiveness to TRAIL therapy is warranted such as biomarkers of autophagosome [4]. The personalized medicine based on the combinational therapy of TRAIL and sensitizer tailored with the biomarker screening of patient's responsiveness towards TRAIL holds a great potential of success for TRAIL targeted cancer therapy in the near future.

\section{References}

1. Wong RS (2011) Apoptosis in cancer: from pathogenesis to treatment. J ExpClin Cancer Res 30: 87.

2. De Miguel D, Lemke J, Anel A, Walczak H, Martinez Lostao L (2016) Onto better TRAILs for cancer treatment. Cell Death Differ 23(5): 733-747.

3. Walczak H, Miller RE, Ariail K, Gliniak B, Griffith TS, et al. (1999) Tumoricidal activity of tumor necrosis factor-related apoptosis-inducing ligand in vivo. Nat Med 5(2): 157-163.

4. Di X, Zhang G, Zhang Y, Takeda K, Rosado LAR, et al. (2013) Accumulation of autophagosomes in breast cancer cells induces TRAIL resistance through downregulation of surface expression of death receptors 4 and 5. Oncotarget 4(9): 1349-1364.

5. Ozoren N, El Deiry WS (2002) Defining characteristics of Types I and II apoptotic cells in response to TRAIL. Neoplasia 4(6): 551-557.

6. Rudner J, Jendrossek V, Lauber K, Daniel PT, Wesselborg S, et al. (2005) Type I and type II reactions in TRAIL-induced apoptosis - results from dose-response studies. Oncogene 24(1): 130-140.

7. Lemke J, Von Karstedt S, Zinngrebe J, Walczak H (2014) Getting TRAIL back on track for cancer therapy. Cell Death Differ 21(9): 1350-1364.

8. Trivedi R, Mishra P (2015) Trailing TRAIL resistance: novel targets for TRAIL sensitization in cancer cells. Front Oncol 5: 69.

9. Dimberg LY, Anderson CK, Camidge R, Behbakht K, Thorburn A, et al. (2013) On the TRAIL to successful cancer therapy? Predicting and counteracting resistance against TRAIL-based therapeutics. Oncogene 32(11): 1341-1350.

10. Thorburn A, Behbakht K, Ford H (2008) TRAIL receptor-targeted therapeutics: Resistance mechanisms and strategies to avoid them. Drg Resist Updat 11(1-2): 17-24.

11. Zhang L, Fang B (2005) Mechanisms of resistance to TRAIL-induced apoptosis in cancer. Cancer Gene Therapy 12(3): 228-237.

12. Abdulghani J, El Deiry WS (2010) TRAIL receptor signaling and therapeutics. Expert OpinTher Targets 14(10): 1091-1108.
13. Horak P, Pils D, Haller G, Pribill I, Roessler M, et al. (2005) Contribution of epigenetic silencing of tumor necrosis factor-related apoptosis inducing ligand receptor 1 (DR4) to TRAIL resistance and ovarian cancer. Mol Cancer Res 3(6): 335-343.

14. Bin L, Thorburn J, Thomas LR, Clark PE, Humphreys R, et al. (2007) Tumor-derived mutations in the TRAIL receptor DR5 inhibit TRAIL signaling through the DR4 receptor by competing for ligand binding. J BiolChem 282(38): 28189-28194.

15. Wagner KW, Punnoose EA, Januario T, Lawrence DA, Pitti RM, et al. (2007) Death-receptor O-glycosylation controls tumor-cell sensitivity to the proapoptotic ligand Apo2L/TRAIL. Nat Med 13(9): 1070-1077.

16. Yoshida T, Shiraishi T, Horinaka M, Wakada M, Sakai T (2007) Glycosylation modulates TRAIL-R1/death receptor 4 protein: different regulations of two pro-apoptotic receptors for TRAIL by tunicamycin. Oncol Rep 18(5): 1239-1242.

17. Merino D, Lalaoui N, Morizot A, Schneider P, Solary E, et al. (2006) Differential inhibition of TRAIL-mediated DR5-DISC formation by decoy receptors 1 and 2. Mol Cell Biol 26(19): 7046-7055.

18. Mohammad RM, Muqbil I, Lowe L, Yedjou C, Hsu HY, et al. (2015) Broad targeting of resistance to apoptosis in cancer. Semin Cancer Biol35: S78-S103.

19. Bolden JE, Peart MJ, Johnstone RW (2006) Anticancer activities of histone deacetylase inhibitors. Nat Rev Drug Discov 5(9): 769-784.

20. Esteller M (2008) Epigenetics in cancer. N Engl J Med 358(11): 11481159.

21. Hopkins Donaldson S, Bodmer JL, Bourloud KB, Brognara CB, Rg Tschopp J, et al. (2000) Loss of caspase-8 expression in highly malignant human neuroblastoma cells correlates with resistance to tumor necrosis factor-related apoptosis-inducing ligand-induced apoptosis. Cancer Res 60(16): 4315-4319.

22. Hopkins Donaldson S, Ziegler A, Kurtz S, Bigosch C, Kandioler D, et al. (2003) Silencing of death receptor and caspase-8 expression in small cell lung carcinoma cell lines and tumors by DNA methylation. Cell Death Differ 10(3): 356-364.

23. Elias A, Siegelin MD, Steinmuller A, Deimling A von, Lass U, et al. (2009) Epigenetic silencing of death deceptor 4 mediates tumor necrosis factorrelated apoptosis-inducing ligand resistance in gliomas. Clin Cancer Res 15(17): 545-5465.

24. Kurita S, Higuchi H, Saito Y, Nakamoto N, Takaishi H, et al. (2010) DNMT1 and DNMT3b silencing sensitizes human hepatoma cells to TRAILmediated apoptosis via up-regulation of TRAIL-R2/DR5 and caspase-8. Cancer Science 101(6): 1431-1439.

25. Wu PH, Chen XM, Liu XQ, He JL, Feng Q et al. (2016) Activation of tumour necrosis factor-related apoptosis-inducing ligand (TRAIL) receptor gene expression following DNA demethylation in placental choriocarcinoma and transformed cell lines. ReprodFertil Dev 28(11): 1844.

26. Moriwaki K, Narisada M, Imai T, Shinzaki S, Miyoshi E (2010) The effect of epigenetic regulation of fucosylation on TRAIL-induced apoptosis. Glycoconj 27(7-9): 649-659.

27. Fröhlich LF, Mrakovcic M, Smole C, Lahiri P, Zatloukal K (2014) Epigenetic silencing of apoptosis-inducing gene expression can be efficiently overcome by combined SAHA and TRAIL treatment in uterine sarcoma cells. Plos one 9: e91558.

28. Zhou W, Feng X, Han Han H, Guo S, Wang G (2016) Synergistic effects of combined treatment with histone deacetylase inhibitor suberoylanilidehydroxamic acid and TRAIL on human breast cancer cells. Sci Rep 6: 28004.

29. Jazirehi AR, Arle D (2013) Epigenetic regulation of the TRAIL/Apo2L apoptotic pathway by histone deacetylase inhibitors: an attractive approach to bypass melanoma immunotherapy resistance. Am J ClinExpImmunol 2(1): 55-74. 
30. Kaminskyyy VO, Surovay OV, Vaculova A, Zhivotovsky B (2011) Combined inhibition of DNA methyltransferase and histone deacetylase restores caspase-8 expression and sensitizes SCLC cells to TRAIL. Carcinogenesis 32(10): 1450-1458.

31. Von Haefen C, Gillissen B, Hemmati PG, Wendt J, Güner D, et al. (2004) Multidomain Bcl-2 homolog Bax but not Bak mediates synergistic induction of apoptosis by TRAIL and 5-FU through the mitochondrial apoptosis pathway. Oncogene 23(50): 8320-8332.

32. Hu H, Jiang C, Schuster T, Li GX, Daniel PT, et al. (2006) Inorganic selenium sensitizes prostate cancer cells to TRAIL-induced apoptosis through superoxide/p53/Bax-mediated activation of mitochondrial pathway. Mol Cancer Ther 5(7): 1873-1882.

33. Liu FT, Agrawal SG, Gribben JG, Ye H, Du MQ, et al. (2008) Bortezomib blocks Bax degradation in malignant B cells during treatment with TRAIL. Blood 111(5): 2797-805.

34. Gillissen B, Wendt J, Richter A, Richter A, Müer A, et al. (2010) Endogenous Bak inhibitors Mcl-1 and Bcl-xL: differential impact on TRAIL resistance in Bax-deficient carcinoma. J Cell Biol 188(6): 851-862.

35. Xu L, Yin S, Banerjee S, Sarkar F, Reddy KB (2011) Enhanced anticancer effect of the combination of cisplatin and TRAIL in triple-negative breast tumor cells. Mol Cancer Ther 10 (3): 550-557.

36. Soria JC, Mark Z, Zatloukal P, Szima B, Albert I, et al. (2011) Randomized phase II study of dulanermin in combination with paclitaxel, carboplatin, and bevacizumab in advanced non-small-cell lung cancer. J ClinOncol 29(33): 4442-4451.

37. Rubinsztein DC, Codogno P, Levine B (2012) Autophagy modulation as a potential therapeutic target for diverse diseases. Nat Rev Drug Discov 11(9): 709-730.

38. Maiuri MC, Zalckvar E, Kimchi A, Kroemer G (2007) Self-eating and selfkilling: crosstalk between autophagy and apoptosis. Nat Rev Mol Cell Biol 8(9): 741-752.

39. White E, Di Paola RS (2009) The double-edged sword of autophagy modulation in cancer. Clin Cancer Res 15(17): 5308-5316.

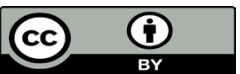

This work is licensed under Creative Commons Attribution 4.0 License

Submission Link: https://biomedres.us/submit-manuscript.php
40. O'Donovan TR, O'Sullivan GC, McKenna SL (2011) Induction of autophagy by drug-resistant esophageal cancer cells promotes their survival and recovery following treatment with chemotherapeutics. Autophagy 7(5): 509-524.

41. Yoon JH, Ahn SG, Lee BH, Jung SH, Oh SH (2012) Role of autophagy in chemoresistance: regulation of the ATM mediated DNA-damage signaling pathway through activation of DNA-PKcs and PARP-1. BiochemPharmacol 83(6): 747-757.

42. Guo XL, Li D, Hu F, Song JR, Zhang SS, et al. (2012) Targeting autophagy potentiates chemotherapy-induced apoptosis and proliferation inhibition in hepatocarcinoma cells. Cancer Lett 320(2): 171-179.

43. White EJ, Martin V, Liu JL, Klein SR, Piya S, et al. (2011) Autophagy regulation in cancer development and therapy. Am J Cancer Res 1(3): 362-372.

44. Degenhardt K, Mathew R, Beaudoin B, Bray K, Anderson D, et al. (2006) Autophagy promotes tumor cell survival and restricts necrosis, inflammation, and tumorigenesis. Cancer Cell 10(1): 51-64.

45. Han J, Hou W, Goldstein LA, Lu C, Stolz DB, et al. (2008) Involvement of protective autophagy in TRAIL resistance of apoptosis-defective tumor cells. J BiolChem 283(28): 19665-19677.

46. Herrero Martin G, Hoyer Hansen M, Garcia Garcia C, Fumarola C, Farkas T, et al. (2009) TAK1 activates AMPK-dependent cytoprotective autophagy in TRAIL-treated epithelial cells. EMBO J 28(6): 677-685.

47. Gillissen B, Richter A, Richter A, Preissner R, Schulze-Osthoff $K$, et al. (2017). Bax/Bak-independent mitochondrial depolarization and reactive oxygen species induction by sorafenib overcome resistance to apoptosis in renal cell carcinoma. J BiolChem 292(16): 6478-6492.

48. Gillissen B, Richter A, Overkamp T, Essmann F, Hemmati P, et al. (2013) Targeted therapy of the XIAP/proteasome pathway overcomes TRAILresistance in carcinoma by switching apoptosis signaling to a Bax/Bakindependent "type I" mode. Cell Death Dis 4: e643.

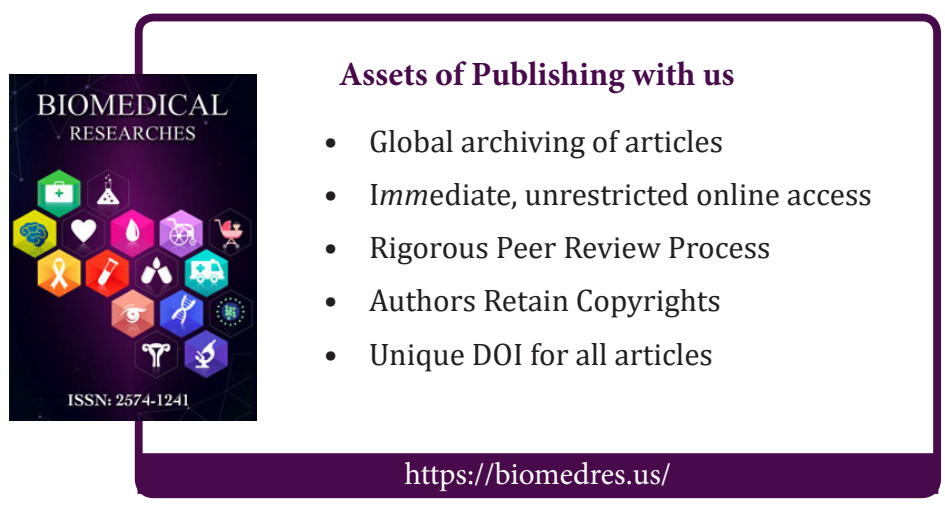

